\title{
A treatable cause of heart failure
}

\author{
Una causa tratable de insuficiencia cardiaca
}

Primary cardiac tumors are extremely rare, and myxomas are the most common primary cardiac neoplasm. Approximately $80 \%$ of myxomas originate in the left atrium, and they may be symptomatic or found incidentally. The cardiovascular manifestations depend on the anatomic location of the tumor. Tumors involving the heart may cause symptoms by obstruction of circulation (producing symptoms of heart failure), interference with heart valves, embolization, direct invasion of the myocardium, invasion of adjacent lung, or by the production of constitutional symptoms. If a cardiac tumor is suspected, imaging procedures are used to determine if a mass is present and where it is located within the heart. The echocardiography is the simplest technique for assessment. Usually myxomas are managed by surgical resection because of the risk of embolization and other cardiovascular complications. ${ }^{1,2,3}$

The authors report the case of a 72-year-old woman with history of type $2 \mathrm{DM}, \mathrm{HT}$ and dyslipidaemia, that was asymptomatic until 3 months earlier, when she reported a 15-kg weight loss. Also, she had been feeling progressively worsening effort dyspnoea for a month and dyspnoea at rest, orthopnoea and paroxysmal nocturnal dyspnoea for 8 days. An outpatient echocardiogram revealed a bulky mass in her left atrium (LA). She was referred to the emergency room, being subfebrile with polypnea, tachycardia and a peripheral saturation of $81 \%$. A new echocardiogram showed a bulky mass with irregular contours $(78 \times 30 \mathrm{~mm})$ in her $L A$, which had its pedicle at the level of the lower fossa ovalis, was mobile with a protrusion to the left ventricle (LV) going past the ventricular middle region and was obstructing the LV entry tract, with an estimated pulmonary artery systolic pressure of $86 \mathrm{mmHg}$. She underwent a myxoma excision and an interatrial septoplasty. Afterwards, she was asymptomatic.

This case highlights that cardiac myxomas, although being benign tumours, may be potentially severe; if diagnosed promptly they are also a treatable and reversible cause of heart failure. The challenge is to ponder the possibility of a cardiac tumor so that the appropriate diagnostic tests can be conducted, since symptoms may mimic other cardiac conditions.

\section{Bibliography}

1. Reynen K. Cardiac myxomas. N Engl J Med. 1995;333:1610-1617.

2. Messina F, Romano P, Crosca S. Atrial myxomas and different clinical presentations. Int $J$ Cardiol. 2016;203:1136-1137.

3. Goswami KC, Shrivastava S, BahI VK, Saxena A, Manchanda SC, Wasir HS. Cardiac myxomas: clinical and echocardiographic profile. Int J Cardiol. 1998;63:251-259.

\section{Diagnosis:}

\section{Cardiac myxoma}

\section{Lúcia M. Brandão, Duarte L. Silva}

Serviço de Medicina. ULSAM, EPE. Hospital de Santa Luzia. Viana do Castelo

Figure. 1. Bulky mass with irregular contours $(78 \times 30 \mathrm{~mm})$ in the left atrium, with pedicle at the level of the lower fossa ovalis, mobile, with a protrusion to the left ventricle going past the ventricular middle region and obstructing the left ventricle entry tract
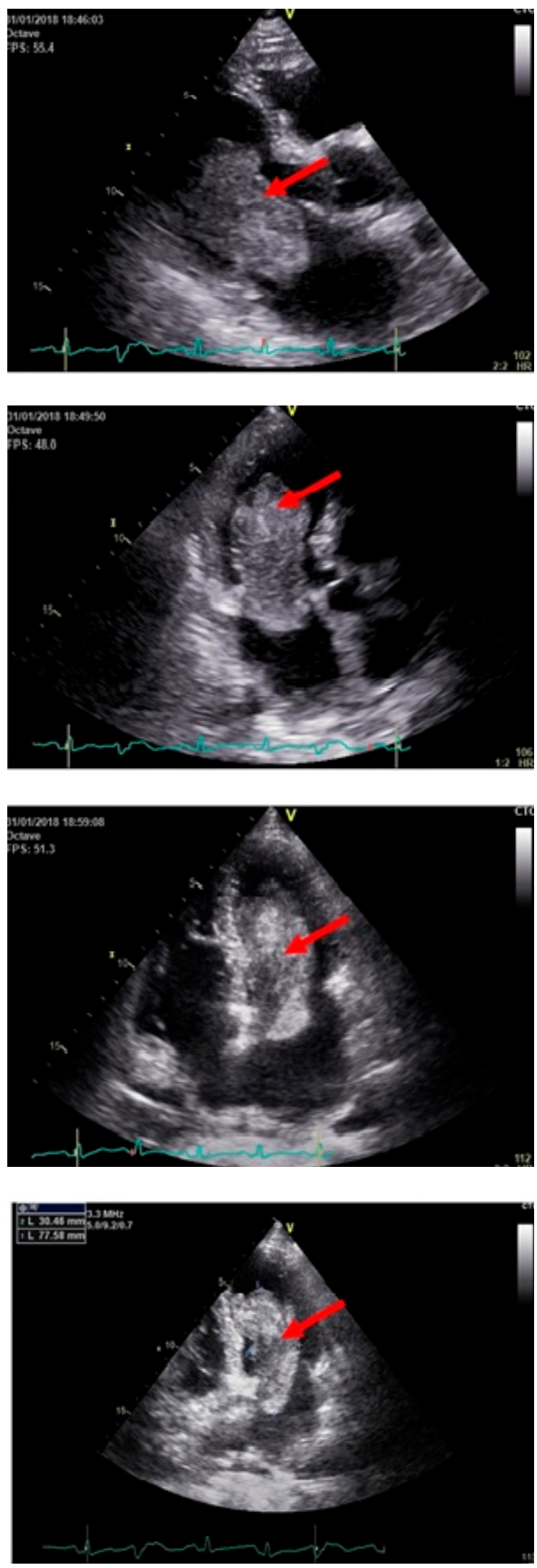